\title{
Radiology update in neuro-ophthalmology
}

\section{Corresponding author:}

John Pula MD

University of Illinois College of Medicine in Peoria

530 NE Glen Oak Avenue

Peoria, IL 61637

Ph 309-655-2164

Fax 309-655-2040

Word count: 2460

\section{KEY POINTS:}

- Both conventional and non-conventional imaging techniques are used in neuroophthlamologic disorders

- MRI is the most commonly used imaging modality in neuro-ophthalmology.

- Particular MRI sequences each have their own advantages in discrimination of different types of lesions.

\footnotetext{
ABSTRACT

Purpose of review

Radiologic imaging is indispensible for the diagnosis and management of many neuroophthalmologic conditions. Advances in the radioimaging of neuro-ophthalmolgic disorders may evolve from the clinical or the radiological side, meaning there is a constant stream of new information for the clinician.

\section{Recent findings}

Functional MRI, diffusion tensor MRI, magnetization transfer imaging, and magnetic resonance spectroscopy are examples of non-standard radiographic techniques which have expanded the knowledge of neuro-ophthalmologic conditions. Studies using conventional MRI have also led to advances in understanding optic neuropathies, the
} 
ocular motor system, pseudotumor cerebri, posterior reversible encephalopathy syndrome and migraine.

\section{Summary}

This article discusses recent radiologic advances relevant to neuro-ophthalmology.

\section{KEYWORDS}

Neuro-radiology, MRI, neuro-ophthlamology

\section{INTRODUCTION}

Imaging techniques for the evaluation of neuro-ophthalmologic disorders include both conventional and specialized types of studies (Table 1). MRI is often the imaging test of choice, but CT has advantages in certain situations, such as in detecting nerve head buried drusen, in the evaluation for orbital decompression in thyroid eye disease, and in detecting bony fracture after orbital trauma or traumatic optic neuropathy.

The language of MRI can be confusing, especially with the variety of possible sequences, but indications for some of these sequences are important to know (Table 2). Intensity is the term used to describe brightness on MRI. T1 weighted images are hyperintense when they reflect water molecules bound to fat or cell membranes, but are hypointense when they reflect free water or tightly bound water (e.g. muscle). T2 weighted images are hyperintense when they represent free water (such as CSF), but become progressively more hypointense as the water becomes more tightly bound. For more detail, an excellent explanation of the physics of MRI from an ophthalmologist's perspective was recently published as a monograph supported by the American Academy of Ophthalmology (1).

\section{OPTIC NEURITIS AND MULTIPLE SCLEROSIS}

Typical MRI features of multiple sclerosis (MS) include disseminated periventricular, callosal, and subcortical white matter T2 signal hyperintensities (figure 1). The McDonald MRI criteria for disease dissemination in space was based on standard Tesla (1.5 T) field strength. Higher field strength MRI magnets (e.g. 3.0 T or greater) are becoming increasingly used. These higher-field MRIs may influence the diagnosis of MS because they detect more lesions and these lesions are more conspicuous with higher field strength MRI (2). On the other hand, higher field strength magnets can also decrease T1 signal contrast. If available, ophthalmologists can request a 3T MRI for patients with optic neuritis at risk for MS, but it is not mandatory that these higher strengths be used.

Other non-conventional MRI techniques are becoming more valuable for imaging MS and optic neuritis (ON). Diffusion tensor (DT) MRI is one such modality. DT MRI applies the direction of water diffusion through tissues to map out neural pathways in the 
brain, such as white matter tracts. This allows imaging based on functional systems (such as the visual system), and can provide insights into rostral changes in the visual neuraxis after damage to first order neurons. DT MRI performed one year after optic neuritis showed changes downstream in the optic radiations, not only in the affected optic nerve (3).

Another MRI technique called magnetization transfer ratio (MTR) can be used to study the central nervous system. MTR images, unlike standard MRI, reflect not only protons on water, but also protons on macromolecules, such as brain tissue. MTR may better reflect the structural integrity of tissue. MTR was studied in optic neuritis, using optical coherence tomography (OCT) and multifocal visual evoked potential (mfVEP) amplitude to differentiate optic nerves with and without axonal loss. When there was MTR reduction in the optic nerve, the OCT retinal nerve fiber layer thickness was decreased and the mfVEP showed decreased amplitude, suggesting that MTR reduction reflects axonal damage rather than demyelination (4). Standard MRI techniques do not always differentiate which T2 MS lesions represent pure demyelination and which reflect axonal loss.

Though DT MRI and MTR hold promise for future study in optic neuritis and MS, ophthalmologists will generally use standard MRI sequences. For the patient with a clinically isolated demyelinating syndrome (CIS) such as ON, the presence and number of disseminated brain lesions on T2 weighted MRI are the most important factors predicting future risk of demyelinating attacks. In contrast, neither retinal nerve fiber layer thinning of the unaffected eye on optical coherence tomography nor a decrease in normalized brain volume on MRI were present in patients after a first attack of optic neuritis (5), suggesting that the first demyelinating event may be too early to find evidence of diffuse axonal degeneration.

The location of MRI lesions in patients with CIS is also important, as having even one brainstem lesion is predictive of an increased risk of conversion to MS and a risk of higher disability compared to patients without brainstem lesions (6). This holds true mainly when there are at least nine lesions. For patients with ophthalmoplegia and CIS who have a visible demyelinating brainstem lesion on MRI, evidence suggests that the ophthalmoplegia improves, and usually resolves, independent of progression to MS (7).

\section{NEUROMYELITIS OPTICA}

Neuromyelitis optica (NMO) is characterized by unilateral or bilateral optic neuritis and longitudinally extensive transverse myelopathy. Figure 2 shows bilateral optic nerve and chiasm involvement in NMO (figure 2). It is usually associated with antibodies to the aquaporin-4 water channel, the NMO antibody. NMO was originally considered as being restricted to the optic nerves and spinal cord, but radiographic brain lesions are now more recognized as the spectrum of NMO expands. In fact, one study showed that $10 \%$ of 
MRIs from NMO patients will have lesions similar in appearance to those of patients with multiple sclerosis (8).

The aquaporin-4 antibody is specific for NMO. A retrospective study in patients with NMO AB positivity showed $79 \%$ had an abnormal MRI, $45 \%$ of which were symptomatic, and $\sim 15 \%$ of which enhanced. These lesions were usually non-specific, but often involve the corticospinal tracts, either in the internal capsule or cerebral peduncle. These structures do not usually show direct damage in MS. MRI lesions in NMO can surround the ventricles. Although periventricular involvement is common in MS, the periventricular involvement in NMO is distinct, appearing as an extensive lesion involving the entire thickness of the corpus callosum, in contrast to the Dawson's fingers of MS (9). Extensive callosal atrophy was similarly reported in a patient with NMO of at least 18 years duration (10). MRI lesions in the area postrema, at the floor of the forth ventricle, may also be characteristic of NMO, but not MS (11). Lesions of the area postrema result in nausea, vomiting, and hiccups, which occur in NMO, but are very rare in MS.

Magnetic resonance spectroscopy (MRS) is an MR technique which measures different brain metabolites, including $\mathrm{N}$-acetyl-aspartate, lactate, choline, and creatine, depending on the tissue being studied. These metabolites increase or decrease from expected norms based on tissue integrity. For example, lactate is elevated in tissue necrosis. MRS was recently studied in NMO. Normal appearing grey and white matter had normal MR spectroscopy markers in NMO. This contrasts to the abnormal MRS values found in MS and marks MRS as a possible future differentiating diagnostic test for NMO against MS (12).

Functional MRI (fMRI) is another non-conventional radiographic test which measures neural activity by measuring changes in cerebral blood flow. Resting state fMRI measurements were different in NMO versus normal controls (13). This difference in functional activity may explain why NMO patients have more cognitive problems than people without NMO (14).

\section{OTHER OPTIC NEUROPATHIES}

Imaging can detect optic nerve abnormalities in many different neuro-ophthalmologic conditions. An example of typical inflammatory optic nerve enhancement is seen in figure 3. Some familiar optic nerve disorders may demonstrate unusual radiographic features on conventional MRI. For example, neuroretinitis can affect the optic pathway beyond the optic nerve head retina, and both intraocular optic nerve and optic nerve sheath enhancement (optic perineuritis) have been reported (15). Similarly, although optic nerve enhancement on MRI usually denotes an inflammatory optic neuropathy, contrast enhancement was also seen in a case of posterior ischemic optic neuropathy due to internal carotid dissection (16). 
Using functional MRI to study cortical reorganization after non-arteritic anterior ischemic optic neuropathy, a correlation was found between visual acuity and activation in the occipital visual areas, suggesting that afferent nerve pathway integrity is related to primary visual cortical activation. Importantly, over time, there is greater activity in the occipital regions when the non-AION eye was stimulated, which speaks to the plasticity of the rostral visual systems after an optic nerve injury. Functional MRI also showed impaired activation in visual pathways after Leber's hereditary optic neuropathy (LHON). Interestingly, radiographic abnormalities in LHON were not restricted to the visual cortex, as the auditory network was also affected (17).

DT MRI may assist in diagnosis of septo-optic dysplasia. Standard T1 and T2 weighted MRI are adequate to show hypoplasia of the optic nerves and abnormalities in midline brain structures. However, by measuring the entire visual tract pathway, DT MRI at higher tesla has the advantage of providing a more comprehensive measurement of the entire visual pathway, and may also allow for earlier radiographic diagnosis of septooptic dysplasia (18).

\section{THE OCULAR MOTOR SYSTEM}

Ferreira et al produced a succinct yet thorough review of the anatomy, pathology and normal MRI characteristics of the ocular motor nerves (19). The brainstem, cisternal, interdural, cavernous, and intraorbital segments are each considered separately. Among the wealth of important and interesting facts in this review, it is mentioned that the ocular motor nerve nuclei and fascicles can not be directly visualized on MRI, and their intracavernous segments can potentially be prone to artifact.

To better detect cranial nerve abnormalities in the cavernous sinus, an MRI technique called contrast enhanced 3D constructive interference in steady-state (CISS) can be used. With superior contrast and resolution, CISS MRI can show smaller structures within the CSF, which makes it ideal for study of cranial neuropathies in the cavernous sinus (20). CISS is not usually included with standard MRI acquisition series, however.

Which ocular motor cases need to be imaged? Savino's group reviewed 93 patients who were older than 50 with no history of cancer and an isolated CN III, IV or VI palsy, and found that only one case had a lesion on MRI to explain the defect (21). The cost and testing exposure to patients does not seem to justify imaging in all patients with ocular motor mononeuropathy. However patients still need to be imaged if the palsy is progressive or persistent. Ophthalmoplegic migraine is one example of an isolated ocular motor neuropathy which may have MRI changes, and because it often affects young people, will often be imaged. In these cases, the third nerve may enhance and enlarge during symptomatic periods, with radiographic resolution during periods of remission (22). 
Trochlear nerve palsy can be congenital or acquired, unilateral or bilateral. Because the trochlear nerve is difficult to visualize (19), it has been historically challenging to confirm that congenital superior oblique palsy is a hypoplastic/aplastic cranial neuropathy, as is the case in congenital third nerve palsy or Duane's syndrome. By using a higher field 3T MRI, patients with congenital superior oblique palsy (SOP) were indeed found to lack a trochlear nerve on the affected side, while visualization of the nerve was present on the contralateral, unaffected side (23). This highly suggests the classification of SOP as a dysinervation disorder. On MRI, the superior oblique muscle is also usually aplastic or hypoplastic in congenital SOP. Controls have almost complete symmetry of the two muscles on MRI, while a wide range of ratios of the thickness of the paretic side to contralateral SO muscle were found in patients with congenital SO palsy, from complete aplasia to equal thicknesses (24). This variability is also found in familial forms of superior oblique hypoplasia (25).

Figure 4 shows a cross section of the ocular motor muscles in the orbit. The presence of supernumerary extraocular muscles (EOMs) has been documented both in cadavers and in vivo, but is difficult to confirm with imaging. When accessory orbital structures are seen on MRI, if they are indeed muscles, they should be isointense to the EOMs. Because Grave's orbitopathy increases the size of these accessory muscles, they may become more obvious on imaging during this clinical state. Since they act as other muscles do, they may enlarge in GO and decrease after treatment (26). Another study concluded that approximately $2 \%$ of humans have deep orbital bands consistent with supernumerary EOMs, which are sometimes significant contributors to restrictive strabismus. Thus, MRI may be helpful in guiding operative management of strabismus (27).

\section{PSEUDOTUMOR CEREBRI}

Imaging of the venous sinuses in pseudotumor cerebri is discussed elsewhere in this edition of Current Opinion in Ophthalmology. The radiologic findings of pseudotumor cerebri (idiopathic intracranial hypertension/IIH) in fact require that brain imaging not show another cause of increased intracranial pressure. That being said, there are some radiologic features of IIH which deserve mention. The commonly described 'slitlike ventricles' is not actually helpful in diagnosing IIH (28). Figure 5 illustrates certain features of IIH that are supportive of the diagnosis, which include an enlarged optic nerve sheath, optic nerve tortuosity, protrusion of the optic nerve head, and concavity or flattening of the posterior globes (29). Two additional MRI features of IIH have now been described: a narrowing of Meckel's cave and a reduction in the diameter of the cavernous sinuses. Narrowing of Meckel's (the trigeminal) cave, which normally appears as CSF signal lateral to the posterior cavernous sinus and clivus on axial sections, is an especially sensitive MRI marker of IIH (30). 


\section{PRES}

Posterior reversible encephalopathy syndrome (PRES) may present to the ophthalmologist as cortical blindness, often associated with mental status changes or seizures. It occurs mainly in the setting of eclampsia, hypertension, chemotherapeutic agents, or sepsis, as a vasculopathy with characteristic MRI abnormalities of bilateral cortical vasogenic edema (figure 6). Because the diseases associated with PRES also can cause coagulopathy, it is rational that one report found the incidence of subarachnoid and intraparenchymal hemorrhage in PRES nears 20\%. The subarachnoid blood appears within the sulci, with sparing of the basal cisterns. This distribution is not found commonly in aneurysmal rupture, and extensive workup for aneurysm in patients with PRES with this pattern of hemorrhage is probably unnecessary (31).

\section{MIGRAINE}

Positron Emission Tomography (PET) scanning is another type of functional imaging which uses radiolabeling to measure regional blood flow. PET is used more often in the clinical setting because the whole body can be imaged for hypermetabolic or hypometabolic activity. Single-photon emission computed tomography (SPECT) is similar to PET, though it measures glucose metabolism rather than blood flow, and tissue resolution is not as favorable (1). PET scanning was used to measure cortical activity as a response to light stimulation in migraineurs. PET imaging found more cortical activation after a light stimulus during migraine headaches compared to between migraine attacks (32). This response on PET suggests that the photophobia of migraine may be linked to impaired sensory modulation and cortical hyperexcitibility.

\section{CONCLUSION}

Although practicing clinicians are not expected to possess the knowledge of a radiologist with regards to imaging modalities used in neuro-ophthalmologic conditions, it is important that they remain up to date on both conventional and non-conventional radiographic techniques.

Critical review of the limits and advantages of imaging allows the ophthalmologist to make informed choices about when to order imaging, which type of scan to order, and how to interpret the results.

\section{REFERENCES}


1) Johnson MC, Policeni BA, Lee AG, Smoker WR. Neuroimaging in ophthalmology, second edition. Oxford University Press, New York, 2011.

* A text reviewing the physical principles behind MRI and other special imaging techniques, along with a description of imaging characteristics of disease.

2) Larsen LS, Larsson HBW, Frederiksen JL. The value of conventional high-field MRI in MS in the light of the McDonald criteria: a literature review. Acta Neurol Scand 2010; 122:149-158.

* Newer MRI sensitivity is reviewed in the context of application to diagnosing multiple sclerosis with the help of neuroimaging.

3) Li M, Li J, He H, et al. Directional diffusivity changes in the optic nerve and optic radiation in optic neuritis. The Britsh Journal of Radiology 2011; 84: 304-314.

* Diffusion tensor imaging as it applies to optic neuritis is studied.

4) Klistorner A, Chaganti J, Garrick R, et al. Magnetisation transfer ratio in optic neuritis is associated with axonal loss, but not with demyelination. Neurolmage 2011; 56:21-26.

* The characteristic patterns of magnetisation transfer as it is used for optic neuritis helps to classify axonal loss as a requisite for abnormal MTR.

5) Kallenbach K, Sander B, Tsakiri A, etal. Neither retinal nor brain atrophy can be shown in patients with isolated unilateral optic neuritis at the time of presentation. Mult Scler 2011; 17:89-95.

* Although the first attack of demyelination usually occurs after disease has been present for some time, this study showed that neither OCT or MRI were sensitive enough to measure signs of axonal degeneration.

6) Tintore M, Rovira A, Arrambide G, et al. Brainstem lesions in clinically isolated syndromes. Neurology 2010; 75:1933-1938.

* Brainstem lesions may signal a more severe progression of demyelinating disease in certain patients.

7) Pula JH, Brock K, Kattah JC. Clinical course of patients with ophthalmoplegia caused by radiographically detectable brainstem demyelination occurring as a clinically isolated demyelinating syndrome. J Neuro-ophthalmol 2011 Published ahead of print.

* Patients with ophthalmoplegia as a first presenting sign of demyelinating disease are studies. For those with a visible lesion on MRI, improvement of the eye movement defect occured, regardless of steroid use, spinal tap characteristics, progression to MS. 
8) Pittock SJ, Lennon VA, Krecke K, et al. Brain Abnormalities in Neuromyelitis Optica. Arch Neurol. 2006;63:390-396.

9) Kim W, Park MS, Lee SH, et al. Characteristic brain magnetic resonance imaging abnormalities in central nervous system aquaporin-4 autoimmunity. Mult Scler 2010; 16:1229-1236.

* The MRIs of patient with the NMO antibody show abnormalities outside the spectrum of the optic nerve and spinal cord.

10) Alikhani K, Lee DH, Kremenchutzky M. Corpus callosum atrophy in a patient with neuromyelitis optica. Can J Neurol Sci 2011; 38:165-167.

* A patient with NMO of long duration had extensive callosal atrophy on MRI, suggesting that this structure may be severely degenerated in NMO.

11) Popescu BF, Lennon VA, Parisi JE, et al. Neuromyelitis optica unique area postrema lesions. Neurology 2011; 76:1229-1237.

* Patients with NMO are more suseptible to area postrema lesions versus patients with MS, and this may be a distiguishing characteristic.

12) de Seze J, Blanc F, Kremer S, et al. Magnetic resonance spectroscopy evaluation in patients with neuromyelitis optica. J Neurosurg Psychiatry 2010; 81:409-411.

* No differences in normal brain were found in patients with NMO when utilizing MR spectroscopy, which contrasts with the abnormalities found in MS.

13) Liu Y, Liang P, Duan Y, et al. Abnormal baseline brain activity in patients with neuromyelitis optica: A resting-state fMRI study. Eur J Radiol 2010, published ahead of print.

14) Blanc F, Zephir H, Lebrun C, et al. Cognitive Functions in Neuromyelitis Optica. Arch Neurol. 2008;65(1):84-88

15) Vaphiades MS, Wigton EH, Ameri H, Lee AG. Neuroretinitis with retrobulbar involvement. J Neuro-Ophthalmol 2011; 31:12-15.

* The authors describe 2 cases where neuroretinitis was associated with abnromalities in the orbit only found by MRI.

16) Kawabe K, Nagaoka T, Iguchi H, et al. Optic nerve MRI enhancement in posterior ischaemic optic neuropathy due to internal carotid artery dissection. Clinical Neurology and Neurosurgery. 2010; 112:350-352.

* An unusual case of optic nerve enhancement in PION. 
17) Rocca MA, Valsasina P, Pagani E, et al. Extra-visual funcional and structural connection abnormalities in Leber's hereditary optic neuropathy. Plos ONE 2011; 6:1-10.

* Functional MRI is used in Leber’s hereditary optic neuropathy.

18) Salmela MB, Cauley KA, Nickerson JP, et al. Magnetic resonance diffusion tensor imaging (MRDTI) and tractography in children with septo-optic dysplasia. Pediatr Radiol 2010; 40:708-713.

* The utility of DTR in DeMorsier's disease is described.

19) Ferreira T, Verbist B, van Buchem $M$, et al. Imaging the ocular motor nerves. European Journal of Radiology 2010; 74:314-322.

* A review article summarizing the radiologic imaging characteristics and patterns of the ocular motor nerves.

20) Yagi A, Sato N, Takahashi A, et al. Added value of contrast-enhanced CISS imaging in relation to conventional MR images for the evaluation of intracavernous cranial nerve lesions. Neuroradiology 2010; 52:1101-1109.

* This article describes CISS imaging as possibly more sensitive to finding lesions in the cavernous sinus to explain clinical ocular motor deficits.

21) Muchison AP, Gilbert ME, Savino PJ. Neuroimaging and acute ocular motor mononeuropathies. Arch Ophthalmol 2011; 129:301-305.

* MRI results from a series of patients presenting with isolated cranial ocular motor nerve palsies finds a low diagnostic yield of MRI within a specific cohort of patients (older than 50, no cancer, no other symptoms).

22) Miglio L, Feraco P, Tani G, Ambrosetto P. Computed tomography and magetic resonance imaging findings in ophthalmoplegic migraine. Pediatr Neurol 2010; 42:434436.

* A case of relapsing-remitting third nerve palsy attributed to ophthalmoplegic migraine shows characteristic enhancement which appears only concomitant to clinical symptoms.

23) Kim JH, Hwang JM. Absence of the trochlear nerve in patient with superior oblique hypoplasia. Ophthalmology 2010; 117:2208-2213.

* The study uses higher tesla MRI to show that in patiens with congenital superior oblique hypoplasia, the trochlear nerve is absent, with the control being that there was visualization of the trochlear nerve on the opposite, unaffected side. 
24) Uchiyama E, Matsuo T, Imai S, Itoshima E. Paretic side/normal side ratios of crosssectional areas of the superior oblique muscle vary largely in idiopathic superior oblique palsy. Am J Ophthalmol 2010; 149:508-512.

* Superior oblique muscle size difference ratio can be used as a maker for trochlear nerve palsy, although a difference is not seen in every case.

25) Kim JH, Hwang JM. MR imaging of familiar superior oblique hypoplasia. Br J Ophthalmol 2010; 94:346-350.

* In cases of familial trochlear nerve palsy, characteristic imaging features are noted.

26) Sinclair NE, Roberts MA, Hourihan MD, et al. Radiologically manifested accessory extraocular muscles in thyroid eye disease. Ophthal Plast Reconstr Surg 2010; 26:286287.

* Because EOMs increase and decrease with untreated and treated thyroid eye disease, supranumory extraocular muscles can be appreciated on MRI in this situation.

27) Khitri MR, Demer JL. Magnetic resonance imaging of tissues compatible with supernumerary extraocular muscles. Am J Ophthalmol 2010; 150:925-931.

* An article describing the utilization of MRI in distiguishing accessory EOMs.

28) Agid R, Farb RI, Willinsky RA, e al. Idiopathic intracranial hypertension: the validity of cross-sectional neuroimaging signs. Neuroradiology 2006; 48:521-527.

29) Brodsky MC, Vaphiades M. Magnetic resonance imaging in pseudotumor cerebri. Ophthalmology 1998;105:1686-9.

30) Degnan AJ, Levy LM. Narrowing of Meckel's Cave and cavernous sinus and enlargement of the optic nerve sheath in pseudotumor cerebri. J Comput Assist Tomogr 2011; 35:308-312.

* In addtion to MRI findings of empty sella, protrusion of the papillilary region, and enhangement perioptic subarachnoid space, two newly proposed MRI features of pseudotumor cerebri are offered.

31) Sharma A, Whitesell RT, Moran KL. Imaging pattern of intracranial hemorrhage in the setting of posterior reversible encephalopathy syndrome. Neuroradiology 2010; 52:855-863.

* PRES patients may have bleeding in the subarachnoid or intraparenchymal brain regions, likely as a consequence of the vasculopathy which causes the syndrome. 
32) Denuelle M, Boulloche N, Payoux P. A PET study of photophobia during spontaneous migraine attacks. Neurology 2011;76:213-218.

* This article highlights the role of PET scanning to determine that photophobia during migraine is probably a response to impaired sensory integration occurring at the level of the brainstem.

\section{Figure Legend.}

\section{Figure 1.}

Typical MRI features of multiple sclerosis. Clockwise from the bottom: sagittal FLAIR MRI showing periventricular ovoid "Dawson's fingers" plaques; T1 axial black holes; T1 post-gadolinium areas of enhancement; sagittal cervical spine MRI showing nonlongitudinally extensive demyelinating lesions.

\section{Figure 2.}

Arrow on the left image shows a normal non-enhancing chiasm on T1 post-gad orbital fat sat MRI. Arrow on the right image shows contrast enhancement to the optic nerves and chiasm of a patient with NMO.

\section{Figure 3.}

MRI in optic neuritis. Top image shows an axial fat-sat contrast enhanced optic nerve OS (arrow). Bottom image shows enhancement of the same left optic nerve on axial sequences (arrow).

\section{Figure 4.}

Normal MRI anatomy of the ocular motor system. Arrowheads clockwise from the top: superior rectus/levater palpebre superioris complex, medial rectus, inferior rectus, lateral rectus; straight arrow: superior oblique; curved arrow: superior orbital vein.

\section{Figure 5.}

MRI abnormalities seen in pseudotumor cerebri. Fat arrowheads: papillary bulging; curved arrow: posterior scleral flattening; thin arrow: optic nerve tortuosity.

\section{Figure 6.}


Posterior reversible encephalopathy syndrome. Axial FLAIR image showing bilateral parieto-occipital hyperintensities. 